\title{
Divulgación científica con participación de estudiantes de Grado en Ingeniería Civil y Máster en Ingeniería de Caminos, Canales y Puertos
}

\section{Jorge Molines Llodrá ${ }^{\mathrm{a} *}$, Patricia Mares-Nasarre ${ }^{\mathrm{b}}$, M. Esther Gómez-Martín ${ }^{\mathrm{c}}$, Vicent Esteban Chapapría ${ }^{d}$}

Departamento de Ingeniería e Infraestructuras de los Transportes, Universitat Politècnica de València

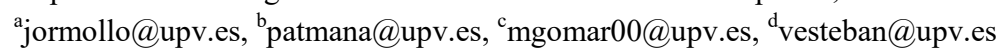

\begin{abstract}
Resumen
El Espacio Europeo de Educación Superior introduce el aprendizaje por competencias como un pilar fundamental en la formación universitaria. Esas competencias incluyen las específicas de la titulación, definidas en el plan de estudios, y las transversales que permiten mejorar la inserción laboral de los estudiantes. La Universitat Politècnica de València (UPV) dispone de un proyecto institucional con 13 Competencias Transversales, las cuales se trabajan y evalúan mediante (1) distintas actividades docentes en asignaturas que son puntos de control, (2) trabajos fin de grado y máster y/o (3) actividades extracurriculares. Sin embargo, algunas de ellas son dificiles de trabajar en el aula. El cono de Dale (1969) o la pirámide de Glasser (1998), indican que aprendemos sólo un 10\% de lo que leemos mientras que ese porcentaje se incrementa a más del 90\% cuando enseñamos a otros. Durante los dos últimos años, se ha desarrollado un taller de Ingeniería Maritima en el Laboratorio de Puertos y Costas dirigido a alumnos entre 15 17 años dentro de la convocatoria CiènciaLab de la UPV. El taller consta de 3 actividades simultáneas y en su última edición han participado en su preparación y desarrollo alumnos de los últimos cursos del Grado en Ingeniería Civil y del Máster en Ingeniería de Caminos, Canales y Puertos. Esta colaboración está en línea con el incremento de aprendizaje que se produce cuando enseñamos a otros y además permite a los alumnos trabajar de primera mano competencias como la de Comunicación Efectiva y la de Planificación y gestión del tiempo, entre otras.
\end{abstract}

Palabras clave: Ingeniería Civil, Competencias Transversales, cono de Dale, pirámide de Glasser, CiènciaLab. 


\section{Introducción}

El Espacio Europeo de Educación Superior (EEES) enfoca el aprendizaje de cualquier titulación universitaria mediante la adquisición de competencias. En el EEES, los estudiantes deben adquirir las competencias generales y específicas de la titulación que vengan definidas en el plan de estudios así como diferentes competencias transversales (CT). Por ejemplo, en el plan de estudios de la titulación "Grado en Ingeniería Civil" de la UPV encontramos la competencia general "Comprender y utilizar el lenguaje propio de la ingeniería así como la terminología propia de la Ingeniería Civil" y la competencia específica "Construir y conservar obras marítimas". Habitualmente, las competencias generales se trabajan y adquieren en diferentes asignaturas a lo largo de la titulación, mientras que las específicas se trabajan y adquieren en determinadas asignaturas. Aquella asignatura en la que se trabajan y adquiere una competencia se denomina punto de control.

Sin embargo, la adquisición de competencias generales y específicas tan solo afecta a la formación técnica del estudiante. En el contexto actual, con una internacionalización de las empresas y equipos multidisciplinares, la formación técnica es tan sólo una de las herramientas que nuestros estudiantes (futuros profesionales) emplearán. Por ello, es fundamental que los estudiantes trabajen y adquieran las CT que van a mejorar su inserción laboral y que van más allá de una formación técnica; el objetivo fundamental es dotar al estudiante de unas herramientas que le permitan desarrollar su esfera social-humana, mejorando sus relaciones interpersonales, resolución de conflictos y trabajo en equipo, entre otras, competencias altamente valoradas por los empleadores, ya que diferencian a unos ingenieros de otros.

Para trabajar las competencias transversales, la UPV dispone de un proyecto institucional, que se describe en la sección 2, que permite evaluar las CT mediante (1) actividades docentes en asignaturas que son puntos de control, (2) trabajos fin de grado y máster y/o (3) actividades extracurriculares. Sin embargo, en muchas ocasiones no es sencillo desarrollar tareas en el aula que se adapten a una correcta evaluación de las CT. Según diferentes estudios pedagógicos (Dale, 1969 y Glasser, 1998), aprendemos hasta un 10\% de lo que leemos mientras que aprendemos hasta un $90 \%$ de lo que enseñamos.

Durante los últimos dos años, el Laboratorio de Puertos y Costas de la UPV ha participado en los talleres de divulgación científica CiènciaLab impulsados por la UPV con la colaboración del Ministerio de Ciencia, Innovación y Universidades y Fundación Española para la Ciencia y la Tecnología. En ellos, alumnos de $3^{\circ}$ y $4^{\circ}$ curso de la Educación Secundaria Obligatoria (ESO) participan en diferentes actividades prácticas para conocer las titulaciones de la UPV. En este estudio, estudiantes del Grado en Ingeniería Civil y Máster en Ingeniería de Caminos, Canales y Puertos se integran en el equipo de profesores 
del taller de CiènciaLab ligado a la ingeniería marítima y titulado “¿Cómo nos protegemos de las olas?". De este modo, los estudiantes han participado tanto en las fases de preparación del taller como en el desarrollo del mismo, trabajando así diferentes CT.

En este trabajo se presenta primero el Proyecto institucional de competencias transversales de la UPV. En segundo lugar se desarrolla el marco pedagógico del estudio. En tercer lugar se presentan las actividades desarrolladas en el taller de CiènciaLab y por último las conclusiones del trabajo.

\section{Competencias Transversales en la UPV}

La UPV (2015) dispone de un Proyecto institucional para trabajar y adquirir 13 CT:

CT-1. Compresión e integración

CT-2. Aplicación y pensamiento práctico

CT-3. Análisis y resolución de problemas

CT-4. Innovación, creatividad y emprendimiento

CT-5. Diseño y Proyecto

CT-6. Trabajo en equipo y liderazgo

CT-7. Responsabilidad ética, medioambiental y profesional

CT-8. Comunicación efectiva

CT-9. Pensamiento crítico

CT-10. Conocimiento de problemas contemporáneos

CT-11. Aprendizaje permanente

CT-12. Planificación y gestión del tiempo

CT-13. Instrumental específica

El Proyecto institucional establece tres niveles de dominio para cada CT:

- $\quad$ Primer Nivel: se desarrolla en los cursos $1^{\circ}$ y $2^{\circ}$ del grado

- Segundo Nivel: se desarrolla en $\operatorname{los} \operatorname{cursos} 3^{\circ}$ y $4^{\circ}$ del grado

- $\quad$ Tercer Nivel: se desarrolla en el máster

Para evaluar el grado de adquisición de CT en cada uno de los niveles se disponen de tres fuentes:

- $\quad$ Planes de estudio: se desarrolla una matriz de competencias transversales donde se identifican las asignaturas puntos de control que deberán generar actividades para recoger evidencias del grado de adquisición de la CT en la Guía Docente.

- Trabajos Fin de Grado y de Máster

- Actividades Extracurriculares 


\section{Marco pedagógico}

La "Pirámide de Aprendizaje" es un grupo de modelos de aprendizaje que relaciona diferentes niveles de retención de conocimientos en función de diferentes mecanismos de aprendizaje. Letrun y Hernes (2018) datan su origen a principios del siglo XIX e identifican su principal inconveniente en el tratamiento de la memoria como un disco duro con diferentes directorios pasivos que son accesibles vía la modalidad de percepción de la información (leída, escuchada, etc.). Letrun y Hernes (2018) indican que esta aproximación simplista no está en línea con las investigaciones de los últimos siglos en el desarrollo de la memoria humana en la que se distinguen tres tipos de memoria: sensorial (retención de información inmediata captada mediante nuestros sentidos), "short-term" (memoria de trabajo con capacidad limitada en la que se guardan y codifican parte de la información obtenida vía sensorial) y "long-term" (memoria que no está codificada como las anteriores y de la que sólo retenemos hechos o conceptos).

Los métodos basados en la "Pirámide de Aprendizaje" como Dale (1969) o Glasser (1999) declaran que aprendemos hasta un $90 \%$ de los conceptos cuando los ponemos en práctica (ver Figura 1) mientras que sólo hasta un 10\% de lo que leemos. Letrun y Hernes (2018) coinciden en que esa es una de las estrategias más efectivas de aprendizaje, principalmente por el efecto de repetición de la información. Sin embargo, es necesario un procesamiento profundo de la información y no la simple repetición para que los conceptos permanezcan en la memoria a largo plazo y por tanto sean útiles para el estudiante.

\section{The Cone of Learning}

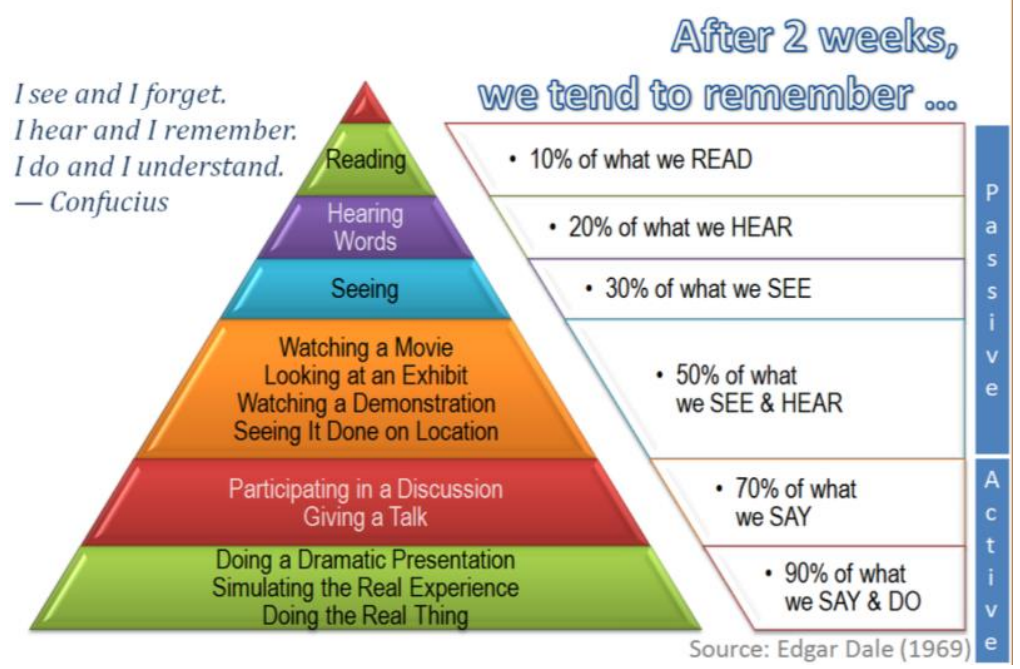

Fig. 1 Pirámide de Aprendizaje (Fuente: Technology and Beyond, 2018) 


\section{Talleres CiènciaLab: ¿Cómo nos protegemos de las olas?}

El taller realizado en CiènciaLab en el Laboratorio de Puertos y Costas de la UPV titulado “¿Cómo nos protegemos de las olas?” acerca a los estudiantes de la ESO a la rama de la ingenería marítima desarrollada dentro de la ingeniería civil. El taller consta de dos etapas desfasadas en el tiempo: Etapa Previa para la adquisición de material y preparación de las actividades y Etapa Ejecutiva en la que se desarrollan las actividades.

Los estudiantes de últimos cursos del Grado en Ingeniería Civil y del Máster de Ingeniería de Caminos, Canales y Puertos se integran desde la Etapa Previa en el desarrollo del taller, donde se les forma sobre las actividades que se realizan y se les prepara para que participen y lideren cualquiera de las cuatro actividades de las que consta la Etapa Ejecutiva. Además, durante la Etapa Previa aportan sus ideas y mejoras sobre las actividades, las cuales se debaten entre el equipo de alumnos y profesores y, si es pertinente, se integran al desarrollo del taller. Estos alumnos universitarios ya han cursado asignaturas específicas de ingeniería marítima y, por tanto, disponen del conocimiento técnico necesario.

Las actividades que se desarrollan en el taller durante la Etapa Ejecutiva tienen una duración máxima de 60 min., y son:

1. Exposición en el aula de conceptos de ingeniería marítima básicos necesarios para comprender mejor las actividades 2, 3 y 4. El contenido son nociones básicas sobre ingeniería de costas y transporte de sedimentos y construcción de obras marítimas de abrigo (diques verticales y en talud) para generar áreas abrigadas del ataque del oleaje. Tiempo estimado 10-15 min.

2. Construcción realista de mantos de diques en talud. En esta actividad, los estudiantes construyen un modelo físico a escala de dique en talud con sus propias manos para comprender mejor la ejecución real de estructuras. Tiempo estimado 15 min. Ver Figura 2 a.

3. Medición de las características de distintos ensayos con oleaje regular en el canal de oleaje 2D del LPC-UPV. En esta actividad, los estudiantes miden la altura de ola, longitud de onda y periodo de oleajes regulares para comprender mejor qué características definen al oleaje (teoría de ondas). Tiempo estimado $15 \mathrm{~min}$. Ver Figura 2 b.

4. Construcción de diques de abrigo para operar una terminal portuaria. En esta actividad, los estudiantes se enfrentan al reto de tener que construir dos obras de abrigo necesarias para proteger una terminal portuaria de la acción del oleaje, de modo que los buques puedan realizar las operaciones de atraque, amarre y carga o descarga en condiciones de seguridad. Tiempo estimado 15 min. Ver Figura 2 c. 
Divulgación científica con participación de estudiantes de Grado en Ingeniería Civil y Máster en Ingeniería de Caminos, Canales y Puertos

La actividad 1 la realizan todos los estudiantes de ESO al mismo tiempo, mientras que las actividades 2, 3 y 4 se realizan paralelamente por grupos de aproximadamente 8 alumnos, de manera que todos los grupos realizan todas las actividades en serie.
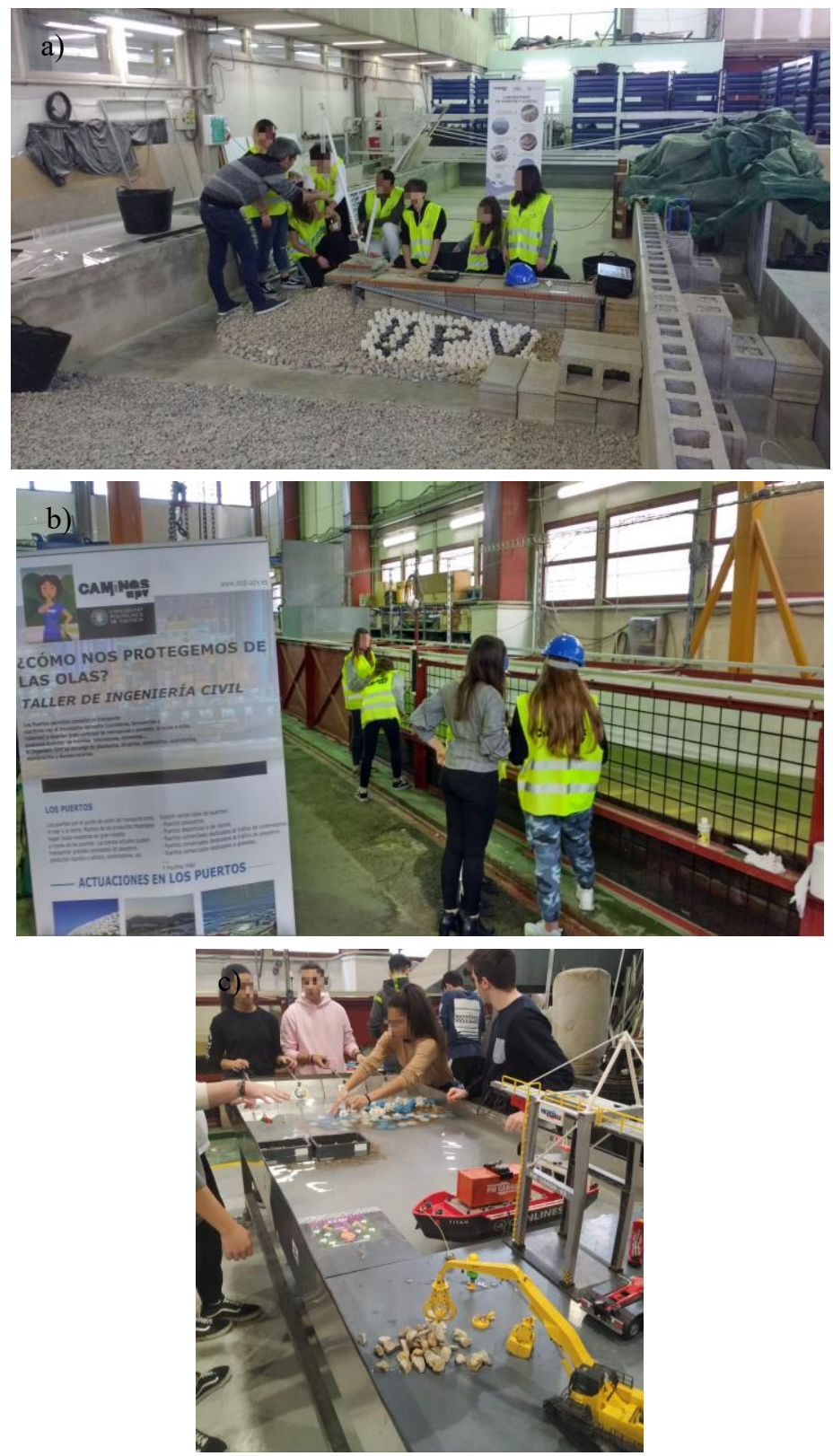

Fig. 2 Actividades del Taller ¿Cómo nos protegemos de las olas? a) construcción realista de diques en talud, b) medición de características del oleaje y c) construcción de diques de abrigo para una terminal portuaria. 
En la Etapa Previa, los estudiantes universitarios trabajan sobre todo laa CTs Aplicación y pensamiento práctico y Trabajo en equipo y liderazgo, mientras que en la Etapa Ejecutiva, asumiendo el rol de profesor y debiendo adaptar el discurso a diferentes audiencias, trabajan sobre todo las CTs Comunicación Efectiva y Planificación y Gestión del Tiempo. En ambas fases, se trabajan también otras CT como son CT Análisis y resolución de problemas (por ejemplo, si alguno de los elementos que emplea pilas deja de funcionar durante la actividad, es necesario aportar soluciones rápidas para el correcto desarrollo del taller) o CT Conocimiento de Problemas Contemporáneos (por ejemplo, este curso 20192020, los estudiantes universitarios debieron responder algunas preguntas relacionadas con el temporal Gloria de enero 2020 y el cambio climático). Los resultados de este trabajo están en línea con los publicados por Carrillo et al. (2018), en los cuales se observa un asentamiento de los conocimientos teóricos aprendidos en clase, así como una mejora en sus habilidades sociales-humanas, por lo que también están trabajando y alcanzando la CT Aprendizaje Permanente.

\section{Conclusiones}

Este trabajo muestra el empleo de talleres divulgativos como técnica complentaria para desarrollar las competencias transversales que deben integrarse dentro del Espacio Europeo de Educación Superior. Los estudiantes universitarios participan en el desarrollo de talleres de divulgación científica a través de los cuales deben poner en práctica conocimientos específicos del plan de estudios y competencias transversales para la correcta consecución del taller.

La colaboración en los talleres por parte de estudiantes del Grado en Ingeniería Civil y el Máster en Ingeniería de Caminos, Canales y Puertos está en línea con el incremento de aprendizaje que se produce cuando enseñamos a otros y además permite a los alumnos trabajar de primera mano algunas de las competencias transversales.

Durante el desarrollo del taller, los estudiantes trabajan su comunicación verbal y no verbal, asientan conocimientos estudiados en clase, planifican y gestionan el tiempo y se adaptan a entornos de trabajo en equipo aprendiendo a debatir constructivamente. Se observa una elevada participacion y motivación del alumnado, de manera que los conocimientos explicados y debatidos seguramente se integren como conceptos asentados en la memoria a largoplazo de los estudiantes universitarios y por tanto tengan un efecto real en su futuro profesional. 
Divulgación científica con participación de estudiantes de Grado en Ingeniería Civil y Máster en Ingeniería de Caminos, Canales y Puertos

\section{Agradecimientos}

Los autores agradecen el apoyo y financiación del Área de Comunicación de la UPV para desarrollar el Taller de CiènciaLab: “¿Cómo nos protegemos de las olas?”.

\section{Referencias}

Carrillo, J.M., Marco, F., García-Bermejo, J.T., Molines, J., Castillo, L.G., (2018). Talleres de Ingeniería Hidráulica dirigidos por estudiantes universitarios. III Congreso Internacional de Innovación Docente, p. 12.

Dale, E., (1969). Audio-Visual Methods in Teaching. $3^{\text {rd }}$ ed., Holt, Rinehart \& Winston, New York, p. 108

Glasser, W., (1999). Choice Theory: A new psychology of personal freedom. HarperPerennial, New York, p. 352.

Letrud, K. and Hernes, S., (2018) Excavating the origins of the learning pyramid myths, Cogent Education, 5:1, 1518638. https://doi.org/10.1080/2331186X.2018.1518638

Technology and Beyond, (2018). Dale's Cone of Experience. [online] Disponible en: https://teachernoella.weebly.com/dales-cone-of-experience.html [Consultado 08 Jun. 2020].

Universitat Politècnica de València, (2015). Competencias Transversales, pp. 95. [online] Disponible en: https://www.upv.es/entidades/ICE/info/Proyecto_Institucional_CT.pdf [Consultado 08 Jun. 2020]. 